\title{
Serodiagnosis of Scrub Typhus cases by Different Diagnostic Tests
}

\author{
Kalpana Mund ${ }^{1}$, Dipti Pattnaik ${ }^{1}$, Shubhransu Patro ${ }^{2}$, J. Jena ${ }^{1}$, \\ N. Singh ${ }^{1}$ and P. Mishra ${ }^{1}$ \\ ${ }^{1}$ Department of Microbiology, ${ }^{2}$ Department of Medicine, Kalinga Institute of Medical \\ Sciences, Bhubaneswar-751024, Odisha, India \\ *Corresponding author
}

\begin{tabular}{|l|}
\hline K e y w o r d s \\
Pyrexia, Scrub \\
typhus, IgM \\
ELISA, Weil-Felix \\
test, ICT
\end{tabular}

\section{Introduction}

Scrub typhus has been one of the most covert emerging and re-emerging Rickettsial infections with increasing trend in incidences of the disease worldwide including India. This tropical febrile vector borne disease also known as "tsutsugamushi disease" is caused by the organism Orientia tsutsugamushi, a gram negative obligate intracellular slow growing bacteria. The infection is transmitted by bite of larval stage (chiggers) of mites belonging to the family Trombiculidae. The
Scrub typhus has been one of the most emerging and re-emerging rickettsial infections with increasing trend in incidences of disease worldwide including India. Clinical diagnosis of scrub typhus is difficult because the signs and symptoms of scrub typhus are almost like other febrile diseases. The mainstay in scrub-typhus diagnosis is serology. In developing countries, among the various laboratory tests to diagnose scrub typhus, WeilFelix test is usually performed despite its low sensitivity. The current study was conducted to compare the efficacy of different serological methods for diagnosis of Scrub typhus. Weil-Felix test, IgM ELISA and ICT were performed in clinically suspected cases of scrub typhus using commercially available kits taking the conventional InBios Scrub Typhus Detect IgM ELISA as reference. Positive Predictive value, Negative Predictive Values, Percentage Sensitivity and Specificity for ICT and Weil-Felix is $100 \%, 98.52 \%, 97.23 \%$, $100 \%$, and $59 \%, 68.71 \%, 23.52,91.22 \%$ respectively. A total of 253 samples were positive in reference standard InBios IgM ELISA. Owing to the constrains of the Weil-Felix test and IFA, commercially available recombinant IgM ELISA and ICT which has a good sensitivity and specificity may be an alternative in laboratories with moderate set up. 
is now prevalent in both rural and urban areas. This might be perhaps due to the migration of people and clearing of forests for building houses, factories etc. (Anitharaj et al., 2016).

Scrub typhus is endemic to a geographically distinct region, the so-called tsutsugamushi triangle, which includes Japan, Taiwan, China, and South Korea. It also occurs in Nepal, Northern Pakistan, Papua New Guinea, and the Australian states of Queensland and Northern New South Wales (Chogle., 2010). During the last two decades outbreak of scrub typhus have been reported from various regions. It was reported from Camp Fuji in 2000 and 2001 (Jiang et al., 2003); from Palau in 2001- 2003 (Durand et al., 2004); from Maldives in 2002 (Lewis et al., 2003); from Asia Pacific region, Australia, Japan, and India in 2003 (Mathai et al., 2003). Serological evidence of scrub typhus has also been reported from Kunduz and Badakhshan provinces of Afghanistan, but no clinical cases have been identified. Scrub typhus has not been reported from Iraq (Endemic inf. Dis., Pp. 51).

Rickettsial diseases have been documented in India since the 1930s with reports of scrub typhus from regions of Kumaon region, Assam in soldiers during the Second World War, from Jabalpur area in Madhya Pradesh and of murine typhus from Kashmir. Scrub typhus cases have been clearly reported from several states in India including Jammu and Kashmir, Himachal Pradesh, Uttarakhand, Bihar, West Bengal, Meghalaya, Rajasthan, Maharashtra, Karnataka, Tamil Nadu and Kerala (DHR ICMR Guidelines, 2015). An estimated one billion people are at risk for scrub typhus and one million cases occur annually. In Tamil Nadu, a region where scrub typhus is endemic, the disease accounts for $50 \%$ of undifferentiated cases of fever presenting to hospital (Roopa et al., 2015). Oreintia is a small ( 0.3 to 0.5 by 0.8 to
$1.5 \mu \mathrm{m})$, gram-negative bacterium of the family Rickettsiaceae. It is endowed with a major surface protein $(56 \mathrm{kDa})$ and some minor surface proteins $(110,80,46,43,39,35$ and $25 \mathrm{kDa}$ ). $O$. tsutsugamushi has many serotypes (Karp, Gillian, Kato, Shimokoshi, Kawazaki and Kuroki) (CD Alert, 2009; Cook GS, 1996).

The vector insect to humans is the larva of trombiculid mites which maintains the infection in nature. There is also a wild rodent reservoir, and the infection characteristically occurs in discrete foci ('mite islands') where infected mites live on the jungle grass Imperfecta cylindrica, known as lalang (Malaysia, Indonesia ), illuk ( Philippines) or kunai ( Papua New Guinea, Australia ), which grows only where the primary jungles has been cleared for cultivation or to build villages. Human cases occurs when workers in oil palm and rubber estates, and police officers and soldiers, traverse this habitat, brushing against the sharp stiff blades of waist-high imperata grass, allowing the larval mites access. It is an important military disease and thousands of cases have occurred in the far East theatre in the Second world War (Cook G S, 1996).

The clinical symptoms are fever, headache, myalgia, malaise, rash and lymphadenopathy which are commonly seen in other acute illness like malaria, enteric fever, leptospirosis, dengue etc. making the clinical diagnosis tough. The pathognomonic clinical sign is "eschar" (cigarette burn like appearance) which is a skin lesion at the site of mite bite and is inconspicuous as it is often present in the genital region and may go unnoticed until looked warily especially in the dark skinned people. Though presence of an eschar alone is sufficient to clinically distinguish scrub typhus from other febrile illness, its presence is highly variable ranging from $10 \%$ to $90 \%$. The treatment is affordable 
and mostly successful with dramatic clinical response to anti-rickettsial drugs within 48 hours (Roopa et al., 2015).

The mainstay in scrub-typhus diagnosis is serology. The oldest test in current use is the Weil-Felix OX-K agglutination reaction, which is inexpensive, easy to perform, and results are available overnight; however, it lacks specificity and sensitivity. The indirect fluorescent antibody (IFA) test is more sensitive, and results are available in a couple of hours; however, the test is more expensive and requires considerable training. IFA uses fluorescent anti-human antibody to detect specific antibody from patient serum bound to a smear of scrub-typhus antigen and is currently the reference standard. Indirect immunoperoxidase (IIP) eliminates the expense of a fluorescent microscope by substituting peroxidase for fluorescein (Koh et al., 2010).

In view of the disadvantages of both IFA and Weil-Felix tests, an alternate serological test, Enzyme Linked Immuno Sorbent Assay (ELISA) is currently the preferred method (Roopa et al., 2015). A rapid diagnosis can be achieved through binding of antibodies that are produced in the serum against the antigen coated in the strip in Immunochromatography test (ICT). Though the sensitivity of the test is high it lacks specificity. PCR is more sensitive as compared to other diagnostic tests. $56 \mathrm{kDa}$ antigen gene is mostly targeted in order to identify the disease (Christopher $e t$ al., 2016).

Although the Scrub typhus disease is endemic in our country, it is grossly underdiagnosed.This is because of non-specific clinical presentation of the disease, lack of access to the specific diagnostic facilities in most areas, and low index of suspicion by the clinicians. So, in this study an attempt has been made to know the seropositivity of scrub typhus in clinically suspected patients by routinely available diagnostic methods.

\section{Materials and Methods}

It is a prospective study which was carried out between October2015 to September 2017, in a tertiary care hospital, Bhubaneswar. The approval for the study was taken from the Institute Ethics committee. Written informed consent was obtained from the patients before enrolment to this study. All the human subjects underwent clinical examination by the clinician for rickettsial and other possible infections. The patients attending this hospital as both inpatients and outpatients who were clinically diagnosed to have typhus fever were included. The patients age varied from 18 to 70 years were taken for the study. The patients who had a history of fever with or without eschar and rash and also had more than 2 symptoms such as headache, myalgia, malaise, nausea, abdominal discomfort were included.

Sera from patients which were positive for any of the following tests like Widal tests, ELISA for dengue, QBC for malaria, rapid test for filariasis and leptospirosis were excluded.

Case definition (DHR ICMR, 2015)

\section{Definition of suspected/clinical case}

Acute undifferentiated febrile illness of five days or more with or without eschar should be suspected as a case of rickettsial infection (if eschar is present, fever of less than five days duration should be considered as scrub typhus). Other presenting features may be headache and rash (rash more often seen in fair persons), lymphadenopathy, multi-organ involvement like liver, lung and kidney involvement. 


\section{Definition of probable case}

A suspected clinical case showing titres of 1:80 or above in OX2, OX19 and OXK antigens by Weil-Felix test and an optical density (OD) $>0.5$ for IgM by ELISA are considered positive for typhus and spotted fever groups of Rickettsiae.

\section{Definition of confirmed case}

A confirmed case is the one in which Rickettsial DNA is detected in eschar samples or whole blood by PCR, or Rising antibody titres on acute and convalescent serum samples detected by indirect immune fluorescence assay (IFA) or IPA.

\section{Specimen collection and processing}

The blood samples were collected from all febrile patients who visited the hospital during the study period and were clinically suspected as typhus fever. Five millilitre of venous blood sample was collected in plain vial from each patient for Weil-Felix test, IgM ELISA and ICT. The study was conducted only after obtaining written informed consent from the patients.

\section{Weil-Felix tube agglutination test}

The test was performed for all samples using the commercial kit (Tulip Diagnostics, Goa ) as per the manufacturer's instructions. Agglutination titres of $\geq 160$ to OX K antigen were considered as positive for scrub typhus.

\section{IgM ELISA for O.tsutsugamushi (Scrub typhus)}

Detection of IgM antibodies by ELISA was carried out using commercial kit (In Bios International, Inc. United states) as per the manufacturer's instructions. The test was standardized with serum samples from healthy blood donors. The cut-off value was obtained by calculating the average of optical density (OD) plus three times of standard deviation (SD) from serum samples of healthy individuals. The $\mathrm{OD} \geq 0.14$ were considered as positive. A set of positive and negative controls were included along with every test reaction.

\section{Rapid ICT}

Rapid test was done using commercially available ICT kit (Mytest Scrub typhus Ab Test Card). Anti-human IgM and Anti-human $\mathrm{IgG}$ antibodies are immobilized on the nitrocellulose membrane respectively. As the test sample flows through the membrane within the test device, recombinant antigencolloidal gold conjugate complexes with specific antibodies (IgM or $\operatorname{IgG}$ ) of O.tsutsugamushi, if present in the sample.

\section{Statistical analysis}

Sensitivity, specificity, PPV and NPV were calculated considering ST IgM ELISA as reference standard.

\section{Results and Discussion}

720 patients suffering from acute febrile illness were subjected for a battery of diagnostic tests such as ICT for malaria, Widal tests for typhoid, ICT for leptospirosis and ELISA for Dengue. 467 samples positive for above mentioned tests were excluded and 253 samples were positive for scrub typhus. $246(34.16 \%)$ were positive for ICT and 100 (13.88\%) samples showed positivity by WeilFelix tests (Table 1). 246 serum samples showed positivity for both ICT and ELISA. However, 7 samples negative for Scrub typhus by ICT were positive by ELISA (Table 2). 59 serum samples showed positivity for both Weil-Felix and ELISA. However, 194 samples negative for Scrub typhus by Weil- 
Felix were positive by ELISA (Table 3). Concordance value of ICT with IgM ELISA of our study is $99.02 \%$ and that of Weil-Felix and IgM ELISA is $69.36 \%$ (Table 4).

Diagnosis of the aetiology of rickettsial diseases can be accomplished most easily and rapidly by demonstrating a significant increase in antibodies in the serum of the patient during the course of infection and convalescence. Several serological tests are currently available for the diagnosis of rickettsial diseases like Weil-Felix Test (WFT), Indirect Immunofluorescence (IIF), Enzyme linked Immunosorbent assay (ELISA) etc.. Although many techniques have been used successfully for rickettsial sero diagnosis, relatively few are used regularly by most laboratories. BSL-3 Lab is not required for performing serological tests (CD Alert, 2009).

Out of 720 serum samples, 253 (35.14\%) were IgM ELISA positive for Scrub typhus followed by ICT 246(34.16\%) and Weil-Felix test 100 (13.88\%). Seropositivity by IgM
ELISA test in the present study correlates well with the findings of Narvencar et al., (2012) (34\%) and Gurung et al., (2013) (30\%). However, Roopa et al., (2015) showed $24 \%$ and Usha et al., (2014) showed (58.21\%) positivity by IgM ELISA. Regarding serodiagnosis by ICT, our finding correlates well with that of Gurung S et al., 2014 31\% but Usha K et al., 2014 showed 57.14\% positivity. Weil-Felix test result of the present study is close to the findings of Rani $\mathrm{S}$ et al., $2016(21 \%)$ but $56.42 \%$ positivity was shown by Usha K et al., 2014.

In the present study $246(34.16 \%)$ samples were positive by both ICT and IgM ELISA and $7(1 \%)$ samples negative for ICT were positive by IgM ELISA. Considering IgM ELISA for Scrub typhus as the reference standard, the sensitivity, specificity, Positive predictive value and Negative predictive value of ICT were $97.23 \%, 100 \%$ and $100 \%$ and $98.52 \%$ respectively. A good correlation exists between the results of ICT and IgM ELISA.

Table.1 Serodiagnosis of Scrub typhus cases by different diagnostic tests

$[n=720]$

\begin{tabular}{|l|c|c|}
\hline TEST & $\begin{array}{c}\text { NO. OF CASES } \\
\text { POSITIVE }\end{array}$ \\
\hline IgM ELISA & 253 & $\%$ \\
\hline ICT & 246 & 35.14 \\
\hline WEIL-FELIX [>1:160] & 100 & 13.88 \\
\hline Total cases=720 & & \\
\hline
\end{tabular}

$246(34.16 \%)$ were positive for ICT and $100(13.88 \%)$ samples showed positivity by Weil-Felix tests

Table.2 Comparison of the results of ICT with IgM ELISA for serodiagnosis of Scrub typhus

\begin{tabular}{|l|c|c|c|}
\hline TEST & ELISA +VE & ELISA -VE & TOTAL \\
\hline ICT+VE & 246 & 0 & 246 \\
\hline ICT -VE & 07 & 467 & 474 \\
\hline TOTAL & 253 & 467 & 720 \\
\hline
\end{tabular}

246 serum samples showed positivity for both ICT and ELISA.

Statistical analysis: Sensitivity: $97.23 \%$. Specificity: 100\%. PPV: 100\% NPV: $98.52 \%$

Concordance: $99.02 \%$ 
Table.3 Comparison of the results of Weil- Felix ( $\geq 1: 160)$ with IgM ELISA

\begin{tabular}{|l|l|l|l|}
\hline TEST & ELISA+VE & ELISA-VE & TOTAL \\
\hline WF +VE $(>1: 160)$ & 59 & 41 & 100 \\
\hline WF -VE & 194 & 426 & 620 \\
\hline TOTAL & 253 & 467 & 720 \\
\hline
\end{tabular}

59 serum samples showed positivity for both Weil-Felix and ELISA.However, 194 samples negative for Scrub typhus by Weil-Felix were positive by ELISA

Statistical analysis: Sensitivity: $23.52 \%$., Specificity: $91.22 \%$, PPV: $59 \%$, NPV: $68.71 \%$.

Table.4 Percentage of overall accuracy (95\% CI)

\begin{tabular}{|c|c|c|c|c|}
\hline TEST & $\begin{array}{l}\text { SENSITIVITY } \\
{[95 \% \mathrm{CI}]}\end{array}$ & $\begin{array}{l}\text { SPECIFICITY } \\
{[95 \% \mathrm{CI}]}\end{array}$ & $\begin{array}{l}\text { POSITIVE } \\
\text { PREDICTIVE } \\
\text { VALUE [95\% } \\
\text { CI] }\end{array}$ & $\begin{array}{l}\text { NEGATIVE } \\
\text { PREDICTIVE } \\
\text { VALUE [95\% } \\
\text { CI] }\end{array}$ \\
\hline ICT & $\begin{array}{l}97.23 \% \\
{[94.38 \% \text { to }} \\
98.88 \%]\end{array}$ & $\begin{array}{l}100 \% \\
{[99.14 \% \text { to } 100 \%]}\end{array}$ & $100 \%$ & $\begin{array}{l}98.38 \% \\
{[96.76 \% \text { to }} \\
99.21 \%]\end{array}$ \\
\hline $\begin{array}{l}\text { WF } \\
\text { titre } \\
\geq 1: 160\end{array}$ & $\begin{array}{l}23.32 \% \\
{[18.25 \% \text { to }} \\
29.03 \%]\end{array}$ & $\begin{array}{l}91.22 \% \\
{[88.28 \% \text { to } 93.03 \%]}\end{array}$ & $\begin{array}{l}59 \% \\
{[49.90 \% \text { to }} \\
67.52 \%]\end{array}$ & $\begin{array}{l}68.71 \% \\
{[67.11 \% \text { to }} \\
70.27 \%]\end{array}$ \\
\hline
\end{tabular}

Comparison between Mytest Rapid kit, Weil-Felix (OX-K) and InBios IgM ELISA ( $\mathrm{n}=253)$

Our finding is close to the findings of Kalawat et al., (2015), Anitharaj et al., (2016). However, study carried out by Gurung et al., (2013) showed rapid ICT positivity in one sample negative by $\operatorname{IgM}$ ELISA. Since ICT also detects IgG antibodies, the patient may have had a secondary infection and thus the positive result in their study.

Our study shows both IgM ELISA and WeilFelix positivity in $59(8.19 \%)$ cases and ELISA positivity was seen in 194 (26.94\%) cases where Weil-Felix was negative. Considering IgM ELISA for Scrub typhus as reference standard, the sensitivity, specificity, Positive predictive value and Negative predictive value of Weil-Felix at a titre of $\geq$ 160 diagnostic for Scrub typhus, is $23.52 \%$, $91.22 \%, 59 \%$ and $68.71 \%$ respectively. Though the sensitivity of Weil-Felix test in our study was low, good specificity and positive predictive value was observed which is close to the findings of Gurung et al., (2013), Anitharaj et al., (2016), Usha et al., (2014). However, good correlation between the results of Weil-Felix test and IgM ELISA was reported by Rani et al., (2016) with sensitivity $72.5 \%$ and Specificity $91.4 \%$.

Weil-Felix results may be negative during the early stages of disease because agglutinating antibodies are detectable only during the second week of illness (Roopa et al., 2015).

Concordance between tests was calculated to have an idea about the agreement between the tests so as to know whether this combination of serological tests can be applied for diagnosing the disease correctly. Concordance value of ICT with IgM ELISA of our study is $99.02 \%$ and that of Weil-Felix and IgM ELISA is $69.36 \% .95 \%$ Confidence interval of ICT in our study is close to IgM ELISA as 
compared to the Weil-Felix test which is similar to the observations of Anitharaj $\mathrm{V}$ et al., (2016). The findings of the present study showed ICT to be a highly sensitive as well as specific test for serodiagnosis of Scrub typhus in comparison to Weil-Felix by taking IgM ELISA as the reference standard. Compared to IgM ELISA, ICT is simpler, easy to perform, rapid and can be used as a screening test for early diagnosis of Scrub typhus in laboratories handling less number of samples. ICT will also help to screen those cases which give the false-positive result by IgM ELISA due to presence of Rheumatoid factor and false-negative results due to secondary infection and rise in IgG level.

In conclusion, our study showed that in the absence of gold standard tests (IFA, IIP and PCR), emphasis should be shifted to early diagnosis with rapid test, Weil-Felix test and ELISA. Rapid ICT kit will help in early diagnosis of Scrub typhus and Weil-Felix test needs to be interpreted in the light of correct clinical context and antibody titres. But, IgM ELISA has good sensitivity and specificity; it is easy to do, gives swift result and is suitable for testing large number of specimens.

\section{Acknowledgement}

We are thankful to all the teaching and nonteaching staffs of the Department of Microbiology and Central laboratory for carrying out the serological tests. Our extended thanks to all the clinicians for their support in sending samples for this study.

\section{References}

Anitharaj V, Stephen S, Pradeep J, et al., Serological diagnosis of acute scrub typhus in southern India: Evaluation of InBios scrub typhus detect $\operatorname{IgM}$ rapid test and comparison with other serological tests. J Clin Diagnostic Res. 2016;10(11):DC07-DC10.

Alert, C.D. Scrub typhus \& other rickettsioses, Vol. 13: No. 1, May-July 2009.

Chogle AR. Diagnosis and treatment of scrub typhus- The Indian Scenario. J Assoc Physician India 2010; 58: 11-2

Christopher S, Suresh A, Dhinakaran Y, Massi C, Sagayaraj IR, P.K G. Scrub Typhus- Epidemiology, Scrub Scrub typhus - epidemiology, pathophysiology, diagnosis, treatment and prophylaxis: a review. J Chem Pharm Sci. 2016; 9(3):1638-1642.

Cook GC, 1996. Manson's Tropical Diseases. 21st Edition. Rickettsial infections sec 7; p 801-906, London: W. B. Saunders Company, Ltd.

DHR ICMR Guidelines for Diagnosis \& Management of Rickettsial Diseases in 2015.

Durand AM, Kuartei S, Togamae I, et al., Scrub typhus in the Republic of Palau, Micronesia. Emerg Infect Dis. 2004; 10(10): 1838-1840.

Endemic infectious diseases of southwest asia; pg 51

Gurung S, Pradhan J, Bhutia PY. Outbreak of scrub typhus in the North East Himalayan region-Sikkim: An emerging threat. Indian $\mathbf{J}$ med Microbiology 2013; 31: 72-74.

Jiang J, Marienau KJ, May LA, et al., Laboratory diagnosis of two scrub typhus outbreaks at Camp Fuji, Japan in 2000 and 2001 by enzyme-linked immunosorbent assay, rapid flow assay, and Western blot assay using outer membrane $56-\mathrm{kD}$ recombinant proteins. Am J Trop Med Hyg. 2003; 69(1): 60-66.

Kalawat U, Ramyasree A, Rani N, Chaudhury A. Seroprevalence of Scrub typhus at a tertiary care hospital in Andhra 
Pradesh. Indian J Med Microbiol. 2015; 33(1): 68.

Koh GCKW, Maude RJ, Paris DH, Newton PN, Blacksell SD. Diagnosis of Scrub Typhus. Am J Trop Med Hyg. 2010; 82(3): 368-370.

Lewis MD, Yousuf AA, Lerdthusnee $\mathrm{K}$, Razee A, Chandranoi K, Jones JW. Scrub Typhus Reemergence in the Maldives. Emerg Infect Dis. 2003; 9(12): 1638-1641.

Mathai E, Rolain JM, Verghese GM, et al., Outbreak of scrub typhus in southern India during the cooler months. Ann N Y Acad Sci. 2003; 990: 359-364.

Narvencar KPS, Rodrigue S, Nevrekar RP, et al., Scrub typhus in patients reporting with acute febrile illness at a tertiary health care institution in Goa. Indian J Med Res. 2012; 136(6): 1020-1024.
Rani S, Thakur K, Sood A et al., Comparison of Weil Felix test and IgM ELISA in the diagnosis of scrub typhus in Kangra, Himachal Pradesh. Int J Health Sci Res. 2016; 6(12): 28-32.

Roopa KS, Karthika K, Sugumar M, Bammigatti C, Shamanna SB, Harish BN. Serodiagnosis of Scrub Typhus at a Tertiary Care Hospital from Southern India. J Clin Diagn Res. 2015; 9(11): DC05-7.

Usha K, Kumar E, Kalawat U, Siddhartha Kumar B, Chaudhury A, Sai Gopal DVR. Seroprevalence of scrub typhus among febrile patients: A preliminary study. Asian J Pharm Clin Res. 2014; 7(SUPPL. 1): 19-21.

Questions FA. Scrub Typhus Scrub Typhus Frequently Asked Questions. World Heal Organ. 2009.

\section{How to cite this article:}

Kalpana Mund, Dipti Pattnaik, Shubhransu Patro, J. Jena, N. Singh and Mishra, P. 2019. Serodiagnosis of Scrub Typhus cases by Different Diagnostic Tests. Int.J.Curr.Microbiol.App.Sci. 8(02): 2145-2152. doi: https://doi.org/10.20546/ijcmas.2019.802.248 